\title{
Techniques de production d'asticots de mouches domestiques (Musca domestica L. 1758) pour l'alimentation des volailles, synthèse bibliographique.
}

\author{
Idriss Hamidou Leyo, Zakari Moussa Ousman, Fréderic Francis \& Rudy Caparros Megido
}

Idriss Hamidou Leyo : doctorant, nationalité Nigérienne. Université Abdou Moumouni de Niamey, Faculté d'Agronomie, Ecole doctorale Science de la Vie et de Terre EDSVT, BP 10960 Niamey, Niger. Auteur correspondant : ihamidouley@outlook.com

Zakari Moussa Ousman : Maître de Conférence, Nationalité Nigérienne. Université Abdou Moumouni de Niamey, Faculté d'Agronomie, Ecole doctorale Science de la Vie et de Terre EDSVT, BP 10960 Niamey, Niger

Fréderic Francis : Professeur, Nationalité Belge. Université de Liège, Faculté Gembloux Agro Bio Tech, Unité d'entomologie fonctionnelle et évolutive, Passage des Déportes 2. 5030 Gembloux, Belgique

Rudy Caparros Megido : Maître de Conférence, Nationalité Belge. Université de Liège, Faculté Gembloux Agro Bio Tech, Unité d'entomologie fonctionnelle et évolutive, Passage des Déportes 2. 5030 Gembloux, Belgique

DOI: $10.25518 / 2295-8010.1813$

\section{Résumé :}

L'alimentation de la volaille nécessite un apport essentiel en protéine. Ces protéines sont introduites aux animaux à travers des ingrédients alimentaires comme les légumes à graines et leurs tourteaux (arachide, soja, etc.), les farines de poisson, etc. Ces sources protéiques bien qu'étant indispensables en alimentation de la volaille, sont assujetties à une flambée de prix et sont peu ou pratiquement non durables. Les asticots des mouches constituent une solution durable en alimentation animale mais sont classées dans les sources méconnues de protéines animales en Afrique de manière générale et au Niger en particulier. La production de ces asticots n'est pas onéreuse et n'implique pas pour le moment une compétition alimentaire avec l'alimentation humaine. Les asticots des mouches domestiques (Musca domestica L. 1758) et des mouches soldats noires (Hermetia illucens L. 1758) sont privilégiés en raison de la facilité de leur production et de l'importance de leur biomasse. Les larves de ces deux types de mouches sont souvent produites avec des déchets de toute nature (substrats) disponibles gratuitement ou cédés dans le cas marchand à un prix dérisoire. Les compositions chimiques de ces asticots sont similaires voire meilleures que celles des ingrédients alimentaires conventionnels utilisés en alimentation avicole, et leur utilisation dans l'alimentation de ces volailles induit de bonnes performances zootechniques aux animaux. En plus de la protéine, le processus de production d'asticots permet d'un coté de recycler les déchets organiques qui constituent un véritable problème environnemental et de l'autre côté il permet de générer un résidu biofertilisant riche en nutriment qui peut être valorisé en agriculture. Plusieurs études ont montré qu'il n'a pas de dans danger sanitaire lié à l'utilisation des asticots en alimentation animale en général et celle de la volaille en particulier. En effet l'évaluation de plusieurs éléments-traces métalliques (ETM) au niveau des asticots avait révélé ces ETM sont à des seuils inferieur à ceux recommandés par 
Techniques de production d'asticots de mouches domestiques (Musca domestica L...

les organismes internationaux (Union Européenne, organisation mondiale de la santé). Quelques rares études ont affirmé qu'une transmission de Salmonelles est possible aux consommateurs. Toutefois il est admis à l'unanimité que les asticots peuvent être introduits en alimentation animal sans induire des effets néfaste sur leur santé et que les contaminations éventuelles sur toute la chaine alimentaire peuvent être contrôlées.

\begin{abstract}
:
Poultry feed requires an essential supply of proteins to be provided to animals through food ingredients such as seed vegetables and their cakes (from peanut, soybean, etc.), fishmeal, etc. These protein sources, although indispensable in poultry feed, are subject to price spikes and are little or practically affordable. Fly maggots are a sustainable solution in animal feed but are classified as one of the little-known sources of animal protein in Africa in general and in Niger in particular. The production of these maggots is not expensive and does not currently involve food competition with human food. The maggots of houseflies (Musca domestica L. 1758) and black soldier flies (Hermetia illucens L. 1758) are preferred because of the easyness of their production and the importance of their biomass. The larvae of both flies are often produced with waste of all kinds (substrates) available free of charge or given away in the commercial case at low price. The chemical compositions of these maggots are similar or even better than those of conventional food ingredients used in poultry feed inducing efficient zootechnical performances to animals. In addition to proteins, the maggot production process allows, on the one hand, to recycle organic waste which is a real environmental problem and, on the other hand, to generate a nutrient-rich biofertilizing residue which can be valorized in agriculture. Several studies have shown that there is no health hazard related to the use of maggots in animal feed in general and on poultry in particular. Indeed, the evaluation of several chemical contaminants in maggots had revealed traces of some molecules at thresholds lower than those recommended by international organizations (European Union, World Health Organization). A few rare studies have affirmed that a transmission of Salmonella is possible to consumers. However, it is unanimously accepted that maggots can be introduced into animal feed without inducing harmful effects on their health and that possible contamination throughout the food chain can be controlled.
\end{abstract}

\title{
Introduction
}

La croissance démographique et l'urbanisation grandissante créent de fortes demandes en produits alimentaires dans le monde qui pourraient être à l'origine d'un déficit important en protéines d'origine végétale et animale (29). Pour éviter une telle situation, d'importants efforts sont consentis afin d'accroître la disponibilité de produits alimentaires. Par exemple, la production de viande de volaille a bondi au niveau mondial, passant de 9 à 120 millions de tonnes entre 1961 et 2016 pour représenter 36\% de la production alimentaire mondiale en 2016 (29). En 2006, l'Afrique de l'ouest comptait plus de 350 millions de têtes de volailles (30) alors que la production moyenne de viande de volaille par an au Niger est passée de 27000 tonnes à quasiment 29000 tonnes entre 2000 et 2007 avant la grippe aviaire de 2008 (61).

L'intensification du secteur avicole, étendue à plusieurs pays africains, est confrontée au manque de disponibilité, à la faible qualité et au coût important des ressources alimentaires protéiques et énergétiques comme les farines de poissons, les farines de légumineuses à graines ou encore les tourteaux d'oléagineux (60). Certaines de ces ressources sont confrontées à une compétition alimentaire avec les autres animaux de rente (e.g. tourteaux de soja) ou à une raréfaction (e.g. 
farines de poissons) entraînant une hausse de leur prix (60). Sur base de ces arguments et vu la contribution de certains insectes à l'alimentation naturelle d'animaux domestiques monogastriques, ces derniers pourraient potentiellement être utilisés comme une alternative aux protéines actuelles d'origine animale et végétale (68). Parmi la diversité de ces insectes, les larves de Diptères (i.e. asticots de mouches) sont les plus plébiscitées de par leur milieu de vie $(38,47)$.

Effectivement, ces dernières sont des décomposeurs de matières organiques et peuvent donc être utilisées pour réduire les déchets organiques générés quotidiennement tout en permettant la production d'aliments pour animaux. Plusieurs espèces de mouches ont été recensées dans la littérature, il s'agit principalement de Fania canicularis (L.) ; Calliphora erytrocephalla (Macquart), Hermetia illucens (L.) ou Musca domestica L.). La plus répandue et la plus connue est la mouche domestique $\left({ }^{*} \mathrm{M}\right.$. domestic**a), fréquemment retrouvée dans les maisons au sein des secteurs urbains ou ruraux. En Afrique de l'Ouest, les asticots de la mouche-soldat noire (H. illucens) et ceux de M. domestica* sont parmi les espèces les plus utilisées dans la production d'asticots pour l'alimentation animale, principalement la volaille $(1,2,14,19,37,41,49,53,64)$. M. domestica est souvent préférée actuellement de par sa prévalence dans la plupart des habitats, son développement rapide et la possibilité d'obtenir des asticots naturellement sur divers substrats sans avoir à élever des mouches adultes pour la ponte (40).

\section{Caractéristiques générales des mouches et des leurs asticots.}

Les Diptères représentent l'un des ordres des insectes les plus importants et les plus diversifiés de point de vue morphologique et biologique. La famille des Muscidae est la famille de mouche la plus répandue dans le monde au sein de laquelle on retrouve les mouches domestiques. En tant qu'insecte holométabole, $M$. domestica passe par quatre stades de développement distincts : l'œuf de couleur blanche, la larve vermiforme et de couleur crème, la pupe de couleur brunâtre et l'adulte ailé de couleur gris-noirâtre (Figure 1 ; (34)). Dans la nature, les adultes de $M$. domestica vivent dans des habitats très variés (34). Les larves ou asticots vivent en milieu terrestre humide où elles se nourrissent de matières végétales ou animales en décomposition (34). En se développant dans les substrats organiques, les asticots participent à la décomposition de ces substrats et permettent leur recyclage.

En tant qu'organismes poïkilothermes, trois paramètres abiotiques essentiels sont à surveiller lors de leur élevage en milieu contrôlé : une température se situant entre 25 à $32^{\circ} \mathrm{C}$, une humidité relative (HR) entre 50 et $75 \%$ ainsi qu'une photopériode pouvant aller de $8 \mathrm{~h}$ à $12 \mathrm{~h}$ de lumière par jour (13). Dans ces conditions d'élevage, les femelles adultes ont démontré une capacité de ponte de plus de 2.000 œufs sur leur durée de vie (un mois en moyenne). La femelle dépose tous les 3 à 4 jours des chapelets d'œufs qui peuvent compter de 75 à 100 unités. Contrairement aux conditions d'élevage au laboratoire, dans les conditions naturelles, une mouche produira environ 500 à 600 œufs au cours de sa vie d'adulte.

Les asticots sont issus des œufs à la surface de matières organiques. En fonction des conditions de température $\left(25\right.$ à $\left.35^{\circ} \mathrm{C}\right)$, l'œuf éclos environ 8 à $12 \mathrm{~h}$ après la ponte (46). Les asticots sont mobiles et s'enfoncent rapidement dans le substrat supportant les œufs. Les asticots de M. domestica ont une morphologie et un mode de vie très différents de l'adulte. La larve est apode, hémicylindrique de couleur crème et acéphale, la tête étant uniquement repérée grâce à la présence de deux crochets buccaux (Figure 1). Les larves matures de M. domestica mesurent de 1,0 à 1,3 cm de long et sont effilées à l'extrémité antérieure et tronquées à l'extrémité postérieure. 
Techniques de production d'asticots de mouches domestiques (Musca domestica L...

Elles se nourrissent presque en permanence pour accumuler des produits de réserve qui serviront à la mue imaginale et sont par conséquent riches en matière de réserve comme les graisses (45).

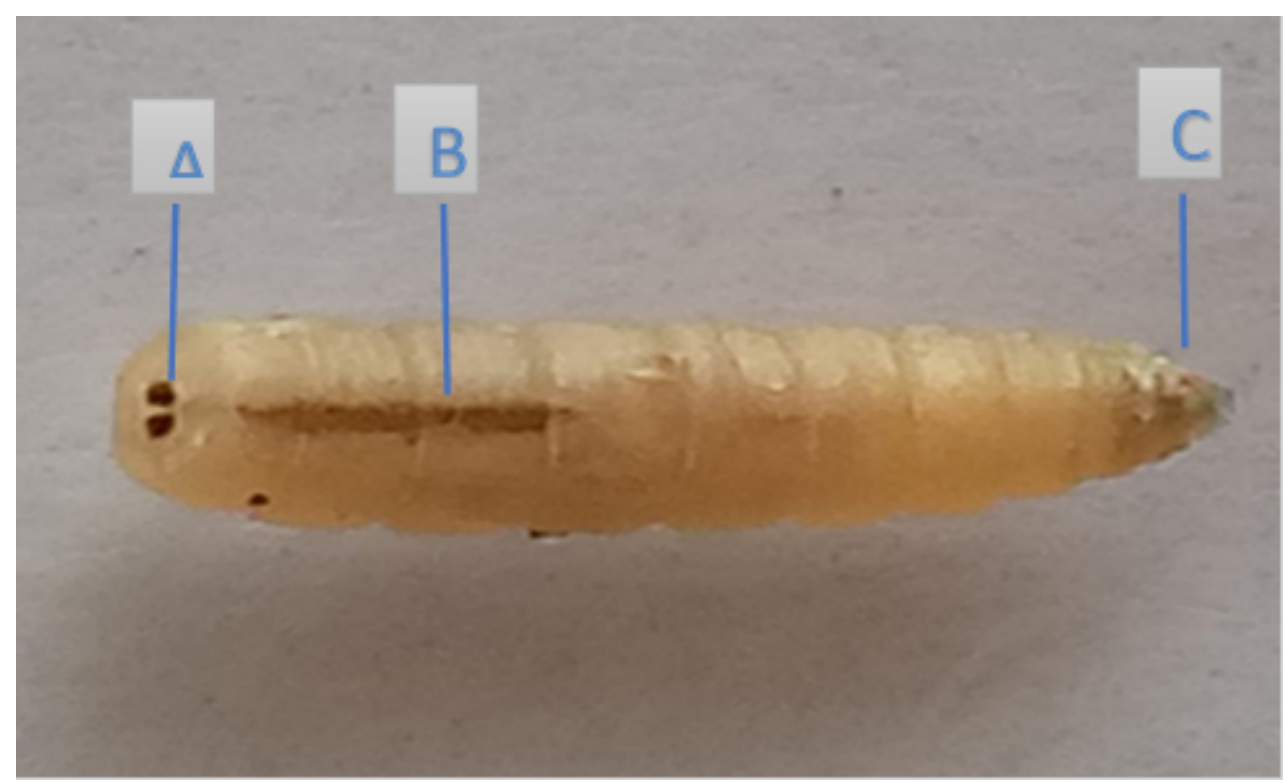

Figure 1 : Asticot de Musca domestica. $A=$ arrière avec des orifices de respiration, $B=$ canaux de respiration, $\mathrm{C}=$ tête. (Leyo 2020).

L'asticot passe par trois stades de développement (L1, L2, L3) appelés stades larvaires (Figure 2). Au premier et deuxième stade larvaire, les larves s'enfoncent dans le substrat car celles-ci préfèrent les milieux humides où elles se nourrissent pendant 3 à 5 jours. Ensuite, la larve du troisième stade accumule les nutriments de réserves nécessaires pour la métamorphose. A la fin de sa période d'accumulation, cette dernière migre vers les milieux plus secs, pour passer au stade pupe. Le poids d'un asticot en fin de maturation varie en général de 0,03 à 0,06 g selon les conditions du milieu (type de substrat, température et humidité) (59). Le stade pupe peut durer 3 à 5 jours, sans s'alimenter et immobile jusqu'à l'émergence de la mouche adulte. 


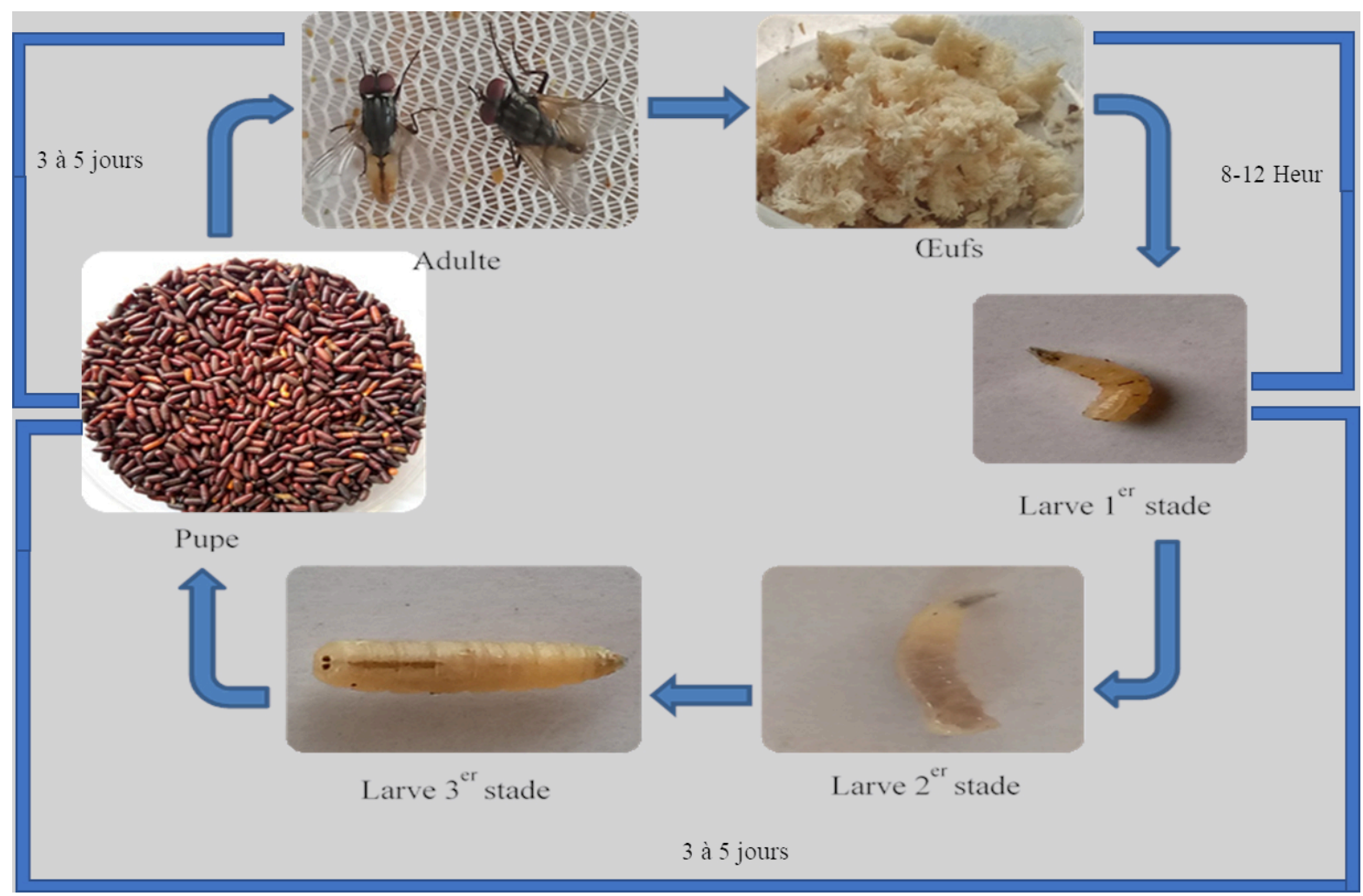

Figure 2 : Cycle de développement de la mouche domestique (Leyo, 2020$).$

\section{Techniques de production d'asticots pour l'alimentation animale}

La production de larves de $M$. domestica peut se concevoir selon deux approches. L'une est traditionnelle avec des investissements quasi inexistants La seconde, semi-industrielle, nécessite des prestations quotidiennes pour laquelle la réduction des coûts de main d'œuvre implique en général des investissements spécifiques voire des aménagements de bâtiments.

\section{Élevage traditionnel}

Lélevage naturel des asticots est simple et peu coûteux puisqu'il requiert des substrats faciles d'accès. Il peut s'agir de déjections animales (poule, vache, porc, lapin ...), de déchets d'abattage ou encore de déchets végétaux (déchets de culture ou ménagers). Toutefois des substrats complémentaires susceptibles de servir dans la production d'asticots avec quelques productivités en biomasse larvaire sont énumérés dans le tableau $1(1,10,25,26,27,37,41,49,52,53,67)$. 
Techniques de production d'asticots de mouches domestiques (Musca domestica L...

Tableau 1 : Diversité des substrats de production d'asticots de Musca domestica

\begin{tabular}{|l|l|l|}
\hline Types & Substrats & $\begin{array}{l}\text { Biomasse } \\
\text { (g/kg de } \\
\text { substrat) }\end{array}$ \\
\hline Animal & $\begin{array}{l}\text { Contenus de rumens additionnés de sang avec ou sans déjections de porcs ; } \\
\text { Excréments de vaches, de volailles, de chevaux ou de porcs ; Excréments de volailles } \\
\text { ou de chevaux additionnés de sang ; Excréments de chevaux additionnés de viscères } \\
\text { de poissons ; Excréments de porcs additionnés de viscères de poulets ; Viscères de } \\
\text { poissons ou de poulets seules ; Viscères de poissons additionnés d'huile de soja. }\end{array}$ & $0,5-160,0$ \\
\hline $\begin{array}{l}\text { Végétal } \\
\text { etêches de brasserie additionnées ou non d'huile de soja ; Son de blé ; Déchets } \\
\text { agricoles ; Résidus des fruits et légumes ; Résidus alimentaires des cuisines }\end{array}$ & $\begin{array}{l}\text { Dréches additionnées de sang ou de contenus de rumens ou des deux ; Drêches } \\
\text { additionnées de viscères de poissons }\end{array}$ & $5,8-94,5$ \\
\hline
\end{tabular}

Source : $(1,10,25,26,27,37,41,49,52,53,67)$.

La technique de production d'asticots en oviposition naturelle est une technique simple et peu chronophage. Il suffit juste de placer les substrats dans des récipients (seau, calebasse, pot ...), de les humidifier à $70 \%$ et de placer le récipient à l'air libre à l'abri du soleil. Les mouches viendront pondre et après trois ou quatre jours, les asticots pourront être collectés par tamisage. Les asticots seront directement distribués aux animaux de rente ou seront mis à mort à l'eau chaude et séchés au soleil pour une conservation durable et une utilisation ultérieure. Une autre technique consiste à verser simplement le contenu des récipients aux animaux de rente comme les volailles qui pourront se nourrir des asticots.

Un certain nombre de paramètres doivent être suivis afin d'optimiser la production d'asticots via cette méthode. Parmi ces derniers, la surface des récipients utilisés est importante car plus cette surface est grande, plus des sites potentiels de ponte (creux et bosses) seront présents et par conséquent la biomasse larvaire sera plus importante. A titre d'illustration, il a été obtenu 127,5 $\mathrm{g}$ de larves en fin de développement (L3) pour $0,07 \mathrm{~m}^{2}$ de surface contre 257,2 $\mathrm{g}$ de larves pour $0,11 \mathrm{~m}^{2}$ (63). Le type de substrat exposé est un facteur d'attraction des différentes espèces de mouches. En effet il a été souligné que $M$. domestica est l'une des mouches les plus actives dans la décomposition des déjections animales (37).

L'utilisation des viscères d'animaux permet quant à elle plutôt d'attirer des mouches de la famille des Calliphoridae (e.g. Chrysomia megacephala (Fabricius, 1794)) que des Muscidae comme M. domestica (50). L'humidité du substrat est un facteur important à contrôler. Une diminution de 99\% de la biomasse larvaire produite sur de la fiente de poule a été observée lorsque l'humidité passait de 60 à plus de $70 \%$. Lorsque l'eau stagne à la surface du substrat, l'oviposition est fortement réduite ainsi que le développement larvaire (63).

Néanmoins, il a été démontré que les stades L3, plus robustes, peuvent accepter des taux d’humidité 
plus importants car l'optimum se situe entre 74 et 78\% d'humidité (67). Finalement, des facteurs abiotiques saisonniers extrêmes comme de fortes températures (e.g. 38-40 $\left.{ }^{\circ} \mathrm{C}\right)$ peuvent avoir un impact négatif sur l'activité des femelles et donc sur la fécondité ainsi que sur l'incubation des œufs et le développement $(19,59,63)$.

Vu l'impact important des facteurs précités, la biomasse des asticots va donc évoluer en fonction des périodes de l'année. Les conclusions des études réalisées en Côte d'Ivoire, au Mali (41) et au Burkina Faso (63) font aussi état de l'effet des saisons et donc des variations climatiques sur la production des larves. Sur base d'un kg de substrat en oviposition naturelle, il a été obtenu $250 \mathrm{~g}$ d'asticots en saison pluvieuse contre $100 \mathrm{~g}$ en saison sèche sur un mélange d'excréments des petits ruminants et de sang; $190 \mathrm{~g}$ d'asticots en saison pluvieuse contre $80 \mathrm{~g}$ en saison sèche sur des excréments de volailles et $300 \mathrm{~g}$ d'asticot en saison pluvieuse contre $50 \mathrm{~g}$ en saison sèche. La sécheresse de la saison influence donc négativement l'incubation des œufs ce qui a pour conséquence une réduction de l'éclosion et le ralentissement du développement des larves (41).

\section{Élevage semi-industriel}

\section{Élevage d'adultes}

L'entretien des colonies d'adultes de $M$. domestica se réalise dans des conditions contrôlées de température se situant entre 25 à $32^{\circ} \mathrm{C}$, d'HR se situant entre 50 et $75 \%$ ainsi que d'une photopériode pouvant aller de 8 à $12 \mathrm{~h}$ de lumière par jour (23). Les adultes peuvent être placés pour la reproduction dans divers volumes allant de petites cages (e.g. 0,03 $\left.\mathrm{m}^{3}\right)$ jusqu'à des pièces de plusieurs $\mathrm{m}^{3}(23,37)$. Pour que les reproductions des adultes soient efficientes dans ces différents volumes, il faudra veiller à contrôler les densités utilisées sur base d'un sexe - ratio d'un mâle pour une femelle qui varient, selon les publications, de 5,6 adultes par $\mathrm{m}^{3}$ à 2,8 adultes par $\mathrm{cm}^{3}(19,23)$. Afin d'accélérer la maturation des organes génitaux, les adultes sont nourris de diverses solutions sucrées dont la valeur nutritionnelle influencera la fécondité (57).

Par exemple, les femelles nourries à l'aide d'une solution d'un mélange de sucre et de lait en poudre (2:1) ont montré des taux d'oviposition 5 fois supérieurs à ceux de femelles nourries d'une solution de sucre et de levure dans les mêmes proportions (19). Finalement, en plus de ces solutions nutritives, il est nécessaire de fournir aux insectes un apport d'eau via un substrat humidifié (e.g. papier filtre ou éponge) $(19,23)$. Trois à quatre jours après l'émergence, les adultes doivent être approvisionnés d'un substrat de ponte pour la collecte des œufs.

Il s'agit de placer au fond de la cage un récipient (e.g. boite de Pétri de $100 \mathrm{ml}$, bout de tissus de 20 $\times 20 \mathrm{~cm}$ ou des coupelles en plastique) contenant un attractant de ponte (e.g. 50-100 g de son de blé fermenté à 60-70\% d'humidité, $50 \mathrm{ml}$ d'excréments de porc ou d'animaux domestiques ou un coton imbibé de lait) qui, par l'odeur qu'il dégage, stimulera la ponte $(1,37)$. Afin de collecter les œufs, un tissu en coton va recouvrir ce récipient et la collecte sera effectuée de manière journalière pendant 5 à 10 jours (23).

\section{Développement larvaire}

Les conditions environnementales de l'unité d'élevage de larves seront similaires à celles de l'unité d'élevage des adultes, à savoir une température entre 25 à $32^{\circ} \mathrm{C}$ et une HR se situant entre 50 et 
Techniques de production d'asticots de mouches domestiques (Musca domestica L...

75\%. Les larves de M. domestica étant des décomposeurs, ces dernières peuvent être élevées sur une grande variété des substrats (Tableau 1).

De manière générale, les substrats de productions peuvent être d'origine animale, végétale ou un mélange des deux à différentes proportions selon la disponibilité des substrats. En principe toutes les matières organiques à décomposition rapide peuvent servir de substrats de ponte et de développement des asticots. Les déchets d'animaux constituent un milieu particulièrement important et probablement le principal pour le développement des larves $M$. domestica vu les biomasses larvaires produites à l'aide des déjections de volailles $(0,5-160,0 \mathrm{~g} / \mathrm{kg}$ de substrats) (Tableau 1). Les résidus végétaux, comme la drêche des brasseries, peuvent également permettre une production en biomasse larvaire et constituent donc des substrats potentiels pour la production d'asticots (Tableau 1).

Toutefois, pour que le développement larvaire soit efficient, la surface du récipient et la quantité du substrat doivent être contrôlées en fonction de la quantité d'œufs de $M$. domestica à incuber (23). Ainsi la densité larvaire pour un développement larvaire efficace est aux alentours de $1 \mathrm{~g}$ d'œufs pour $1 \mathrm{~kg}$ de substrat $(19,23)$. Cependant, il faut estimer ces densités larvaires pour les différents substrats de production et appréhender également les besoins nutritifs des larves pour en améliorer la production.

\section{Techniques de collectes des larves}

Les asticots peuvent être auto-récoltés au cours de leur dernier stade de développement. Puisqu'elles cherchent un endroit sec et humide pour la pupaison, des méthodes pour encourager les asticots à migrer vers un autre milieu facilitant la collecte peuvent être envisagées. Un autre moyen de collecte est la méthode de criblage. Cette méthode consiste à placer au soleil un tamis d'une maille de $3 \mathrm{~mm}$ sur un bassin de récupération, le substrat contenant les asticots est répandu en une fine couche sur le tamis et les asticots tentant d'échapper à la lumière du soleil passent à travers les mailles du tamis pour tomber dans le bassin de récupération (64).

Par ailleurs pour améliorer la technique de collecte par criblage, un dispositif d'extraction des asticots (Figure 3) a été mis au point. Le dispositif n'a pas besoin de la présence de l'exploitant quand le substrat est chargé sur le dispositif et ce dernier peut venir récupérer le produit final qui l'attend dans le réceptacle (60). Toutefois la récolte des asticots semble difficile à un âge précoce et la biomasse reste faible. Par conséquent, il est conseillé de récolter les asticots à un âge avancé (4 jours) pour une biomasse plus importante et une récolte plus facile (8). 

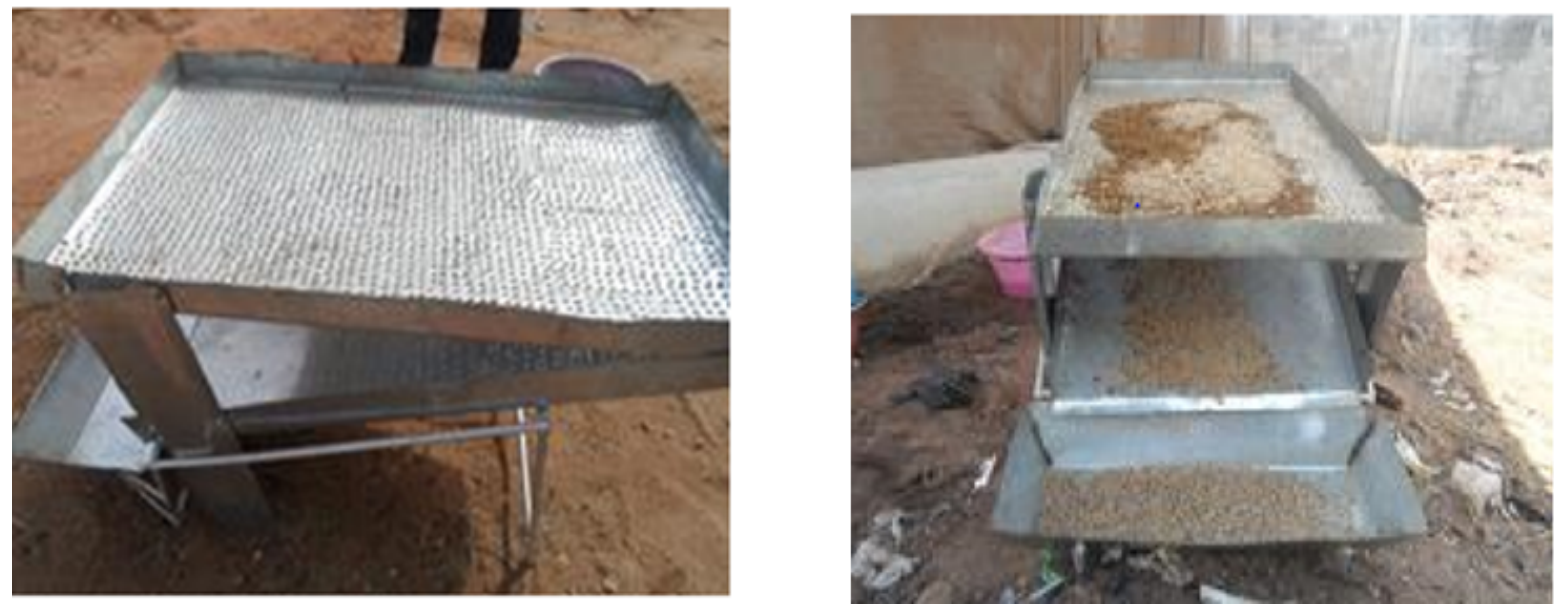

Figure 3 : Extracteur des asticots de mouches domestiques (60).

\section{Valeur nutritionnelle des asticots}

La production d'asticots à l'aide de déchets humains en vue d'une utilisation des protéines et graisses en nutrition animale a été proposée par Lindner en 1919. Depuis lors, des études sont menées pour caractériser la valeur nutritionnelle des asticots et des pupes nourris sur différents milieux de production. Lâge des larves collectées est un déterminant important de leur qualité nutritionnelle. En effet, la teneur en protéines des larves transformées en farine après séchage passait de 55,4\% pour les larves de deux jours à respectivement $50,2 \%$ et 47,1 \% pour celles de trois et quatre jours.

Concernant les lipides, ces derniers augmentent de quelques pourcents (de 20,8 à 25,3 \%) avec l'âge des larves (8). La teneur en macronutriments des pupes peut, quant à elle, être supérieure à celle des larves oscillantes entre 61,0 et $76,0 \%$ pour les protéines et entre 9,0 et $15,5 \%$ pour les lipides en fonction des substrats de développement (59).

En plus de l'âge des larves à la collecte, d'autres paramètres, comme les substrats de croissance utilisés, peuvent influencer la qualité nutritionnelle des asticots produits (68). Il a été démontré que des asticots de $M$. domestica produits sur un mélange de son de blé et de sang de bovin contenaient, en matière sèche, $47,1 \%$ de protéines et $7,5 \%$ de lipides alors que des asticots produits sur des déchets de produits laitiers contenaient quant à eux respectivement $60,0 \%$ et 20,0 \% de protéines et de lipides (9). La méthode de séchage des asticots a également son importance (10).

Ces mêmes auteurs ont obtenu des asticots séchés au four possédant une teneur moyenne en protéines plus élevée (50,9\%) que des asticots séchés au soleil (47,0\%). Du coté des teneurs en lipides, les asticots passent de 22,8 \% pour un séchage au four à 26,4 \% pour un séchage au soleil.

Finalement, la méthode utilisée pour déterminer les nutriments peut avoir un impact sur le résultat obtenu. Par exemple, la méthode utilisée pour déterminer les protéines dans les études précitées était celle de Kjeldahl avec un facteur de conversion du taux d'azote de 6,25. Certains chercheurs (39) ont démontré que le facteur de conversion $(6,25)$ surestimait la teneur en protéines chez les insectes et ont suggéré l'utilisation d'un facteur de 4,76.

De manière résumée et en dépit des paramètres influençant la composition des asticots évoqués 
Techniques de production d'asticots de mouches domestiques (Musca domestica L...

précédemment, la teneur en protéine brute des asticots varie entre 40,0 et 63,0 \% (MS) alors que la teneur en lipides semble quant à elle encore plus variable et se situe entre 9,0 et 26,0 \% (MS). La teneur de ces asticots en cendre varie de 6,2 à 17,3\% (MS) alors que celle en fibre varie de 1,6 à $8,6 \%(2,3,9,28,38,53,54,55,56,59)$.

De la même manière que le substrat de croissance influence les teneurs en macronutriments des asticots, il influence également le profil en acide gras. Ce dernier semble être dominé par l'acide palmitoléique, l'acide oléique et l'acide linoléique (Tableau 2). Par exemple, les larves nourries de lait en poudre, de sucre et de déjections de poules pondeuses ont montré un profil en acides gras composés de 25,0\% d'acide palmitoléique, 16,4\% d'acide linoléique, 21,7\% d'acide oléique (38), tandis que les larves nourries sur le fumier de porc ont montré un profil d'acide gras de 29,1\% d'acide palmitoléique ; 23,3\% d'acide oléique ; 17,2\% d'acide linoléique et 1,3\% d'acide linoléique (70). Concernant le profil en acides aminés, ce dernier est particulièrement dominé par de la lysine 5,0 à 8,2 g/ $100 \mathrm{~g}$ de protéines brutes. Les teneurs en différents minéraux peuvent également fortement varier en fonction des substrats de développement, ils semblent être fortement dominés par le Zn et le Mn. (Tableau 2). 
Tropicultura 2295-8010 Volume 39 (2021) Numéro 2, 1813

Tableau 2 : Profil en acides aminés et en acides gras des asticots de M. domestica 
Techniques de production d'asticots de mouches domestiques (Musca domestica L...

\begin{tabular}{|c|c|c|c|}
\hline Acides aminés & $\%$ & Acides gras & $\%$ \\
\hline \multirow[t]{2}{*}{ Acide aspartique } & $4,50-8,50$ & Acide tridecanoique & 0,10 \\
\hline & & C13 :0 & \\
\hline \multirow[t]{2}{*}{ Acide Glutamique } & $8,90-15,3$ & Acide myristique & $2,00-5,50$ \\
\hline & & $\mathrm{C} 14: 0$ & \\
\hline \multirow[t]{2}{*}{ Alanine } & $4,40-76,0$ & Acide myristoléique & 0,01 \\
\hline & & $\mathrm{C} 14: 1$ & \\
\hline \multirow[t]{2}{*}{ Arginine } & $3,70-5,80$ & Acide pentadécanoïque & 0,40 \\
\hline & & C15 :1 & \\
\hline \multirow[t]{2}{*}{ Cystéine } & $0,50-1,0$ & Acide palmitique & $29,10-32,40$ \\
\hline & & C16 :0 & \\
\hline \multirow[t]{2}{*}{ Glycine } & $3,70-5,1$ & Acide palmitoléique & $17,10-17,40$ \\
\hline & & C16 :1n9 & \\
\hline \multirow[t]{2}{*}{ Histidine } & $1,00-3,60$ & Acide heptadécanoïque & 0,50 \\
\hline & & $\mathrm{C} 17: 1$ & \\
\hline \multirow[t]{2}{*}{ Isoleucine } & $2,30-3,70$ & Acide stéarique & $3,40-3,80$ \\
\hline & & C18 :0 & \\
\hline \multirow[t]{2}{*}{ Leucine } & $4,50-6,40$ & Acide oléique & $21,80-23,30$ \\
\hline & & C18 :1n9 & \\
\hline \multirow[t]{2}{*}{ Lysine } & $5,00-8,20$ & Acide linoléique & $16,40-17,20$ \\
\hline & & C18 :2n6 & \\
\hline \multirow[t]{2}{*}{ Méthionine } & $1,30-3,70$ & Acide $\alpha$-linolénique & $1,30-2,00$ \\
\hline & & $\mathrm{C} 18: 3 \mathrm{n} 3$ & \\
\hline \multirow[t]{2}{*}{ Phénylalanine } & $3,70-5,90$ & Acide $\gamma$-linolénique & 1,99 \\
\hline & & C18 :3n6 & \\
\hline Proline & $2,50-4,70$ & Acid arachidique & 0,09 \\
\hline
\end{tabular}


C20 :0

Sérine

$2,60-3,90$

Acide gondoïque

0,60

$\mathrm{C} 20: 1$

Thréonine

$2,00-4,10$

Acide homo-g-Linolénique

0,59

$\mathrm{C} 20: 3 \mathrm{n}-6$

Tryptophane

$1,40-1,50$

Acide arachidonique

0,10

C20 :4n6

Tyrosine

$2,90-7,10$

Acide érucique

0,05

C22 :1 n-9

Valine

$1,30-4,90$

Acide nervonique

0,03

$\mathrm{C} 24: 1$

Sources : $(9,28,35,38,53,55,70)$

\section{Taux d'incorporation d'asticots dans la nutrition de la volaille}

Du côté de l'alimentation de la volaille, il faut retenir que les essais actuels d'incorporation de farine d'asticots de $M$. domestica n'ont montré aucun effet délétère sur la consommation d'aliments par la volaille $(28,56)$ Néanmoins, il est important de connaître les taux de substitution maximum sans réduction de croissance chez la volaille. Différents taux d'incorporations de farine d'asticots ont été testés $(5,5 \%, 8,0 \%, 11,0 \%)$ chez les éleveurs des canards. Des taux d'incorporation supérieurs à $11,0 \%$ entraînent généralement une diminution de la prise alimentaire et des performances des volailles qui pourrait être liée à la couleur foncée de l’aliment.

\section{Performances zootechniques des volailles nourries à l'aide d'asticots}

Concernant les performances des volailles nourries aux asticots, un gain de poids quotidien, une réduction de la mortalité et un meilleur rendement carcasse sont constatés selon les taux d'incorporation $(5,38,56)$. Certaines études avaient remplacé la farine de poisson par de la farine d'asticots chez des poulets de chair âgés de 0 à 5 semaines et ont rapporté que la farine d'asticots pouvait remplacer jusqu'à 33\% la farine de poisson sans effet négatif sur la consommation d'aliments et la prise de poids des poulets (11). En outre il faut signaler également un rendement économique plus élevé lorsqu'on remplace la farine de poisson par la farine d'asticots.

Par ailleurs un remplacement de 0-100\% de graines de maïs par un mélange d'épluchures de manioc séché et farine d'asticots a montré que ce mélange pouvait remplacer à 50,0\% les graines de maïs sans induire des effets négatifs chez la volaille et permet également une réduction de coût des céréales destinées à la volaille (2). Le taux de 11,0 \% d'incorporation de farine d’asticots 
Techniques de production d'asticots de mouches domestiques (Musca domestica L...

permet d'obtenir de bons résultats de croissance pondérale des canetons et une réduction de leur taux de mortalité (moins de 6 \%) (47). Pour plus d'informations, une vue globale sur l'incorporation des insectes en alimentation de la volaille a été effectué (48).

\section{Aspects sanitaires de la production et l'utilisation des asticots pour l'alimentation de la volaille}

Les insectes peuvent bio accumuler, en plus des composés naturellement présents dans les aliments dont ils se nourrissent, des composés présents dans leur environnement comme les élémentstraces métalliques (ETM), les pesticides ou tout autre élément toxique (17). Le risque chimique lié à la production d'asticots de diverses espèces de Diptères (dont Musca domestica) élevés sur divers déchets (fumier de volaille ou de porc ainsi qu'un mélange de fumier de volaille et de déchets d'aliments pour poisson) a été évalué au Royaume-Uni, en Chine, au Mali et au Ghana (18). Parmi les contaminants potentiels, des traces de produits vétérinaires et phytosanitaires ainsi que des éléments-traces métalliques (ETM), de dioxines, de polychlorobiphényles, d'hydrocarbures aromatiques polycycliques et de mycotoxines ont été recherchées.

Les asticots de $M$. domestica possédaient des niveaux en ETM inférieurs aux concentrations maximales recommandées par la Commission européenne, l'Organisation mondiale de la santé et le Codex alimentarius à l'exception du Cadmium. Cette accumulation en ETM, comme le cadmium ou le plomb immobilisé dans l'exosquelette, a également été mise en évidence dans d'autres études portant sur H. illucens (24).

De manière plus spécifique, la bioaccumulation et le transfert trophique du cuivre $(\mathrm{Cu})$, du zinc ( $\mathrm{Zn})$, du fer ( $\mathrm{Fe}$ ), du nickel (Ni), du cadmium ( $\mathrm{Cd}$ ) et du chrome ( $\mathrm{Cr}$ ) ont été évalués de la production d'asticots de M. domestica jusqu'à la chaîne alimentaire. En effet, ces ETM ont été introduits dans les déchets de cuisine destinés à nourrir les larves de $M$. domestica pour l'alimentation des tilapias.

La biodisponibilité de ces ETM dans les larves était inférieure à celle des déchets alimentaires de base. Les concentrations d'ETM dans les larves de $M$. domestica nourries avec des déchets alimentaires étaient conformes aux normes d'alimentation animale en vigueur en Chine et dans l'Union européenne (UE). Les concentrations de $\mathrm{Cu}$, $\mathrm{Cr}$ et $\mathrm{Cd}$ chez les tilapias nourris avec des aliments commerciaux étaient plus élevées que chez les tilapias nourris avec des larves de $M$. domestica séchées.

Par conséquent, les larves de mouches pourraient accumuler des ETM présents dans les déchets alimentaires pour répondre à leurs besoins nutritionnels et pourraient excrétés les éléments excédentaires. Ainsi les résultats des évaluations des risques pour la santé des consommateurs ont montré que, entre autres, l'ingestion de tilapia nourri avec des larves $M$. domestica ne présentait pas de risque pour leur santé (31).

En plus d'un contrôle qualité des substrats de développement des insectes, des normes adéquates de traitements, de manipulations et de stockage des insectes produits sont nécessaires afin de prévenir toute contamination ou détérioration des produits et afin d'assurer la sécurité et la qualité de l'alimentation animale (62). En effet, des taux d'humidité supérieurs à $5 \%$ dans des farines d'asticots entraînaient le développement de microorganismes et que l'utilisation d'emballages scellés dans des zones de stockage appropriée devait être préconisée (12). 
Actuellement peu d'études ont été réalisées sur l'effet de l'incorporation de farine de $M$. domestica sur la santé du système digestif d'animaux d'élevage. Les effets néfastes sur la santé intestinale de la grenouille-taureau Rana (Lithobates) catesbeiana (Shaw 1802) ont été démontrés lorsque les taux de substitution de la farine de poisson par de la farine de $M$. domestica atteignaient les $75 \%$ (44). Récemment, une étude a démontré que le remplacement total de la farine de poisson par de la farine enrichies en acides gras d'asticots (H. illucens) n'a pas affecté les paramètres de croissance et le statut immunitaire de Tilapia du Nil (4). Chez les poulets de chaires, il a été également démontré une augmentation de la masse du foie et du gésier lorsque les taux d'incorporation des insectes passaient de 50 à 100\% (65). Aussi, la présence de dommages histologiques et pathologiques a été observé chez des rats ayant été nourris avec une alimentation incorporant $10 \%$ de farine d'asticots (espèce non déterminée) (14).

Alors qu'il a été démontré une augmentation des fonctions immunitaires chez les souris Kunning ayant reçu de la farine à base d'asticots dans leur alimentation à différents taux d'incorporation (43). Alors que les paramètres zootechniques des animaux d'élevage précités restent stables, les études présentées semblent montrer que des taux de substitution important (75-100\%) des farines animales habituellement utilisée par de la farine de $M$. domestica dépassant les $50 \%$ induisent des problèmes de santé au niveau du système digestif des animaux d'élevage. A la lecture de ces résultats parfois contradictoires, il apparaît clairement que l'incorporation d'asticots en alimentation animale devra faire l'objet d'études en fonction de l'espèce animale étudiée ainsi que de l'espèce de Diptères incorporée. Une revue globale sur ce domaine de recherche a été publiée récemment (51).

Un élément sanitaire important en lien avec la production de Diptères sont les potentielles nuisances causées par les insectes adultes. En effet, $M$. domestica et d'autres espèces de mouches sont connues pour transporter et transmettre de nombreux agents pathogènes mais également pour occasionner une gêne faciale causant une perturbation dans leur alimentation et donc des pertes de productivité chez les animaux d'élevage $(32,17)$.

De plus, des myiases intestinales peuvent se développer suite à l'ingestion d'œufs de Diptères. Les larves en résultant atteignent le tractus digestif et sont évacués par les fèces. D'une manière générale, ces infections sont asymptomatiques. M. domestica, est rarement rapportée comme une cause de myiase alors que d'autres Diptères sont des agents reconnus comme Chrysomya putoria (Fabricius 1794), récemment utilisée en nutrition du Tilapia (4).

\section{Impact environnemental de la production de Musca domestica}

La production de farine protéinée n'est pas le seul avantage de la production de $M$. domestica puisque les asticots permettent également un recyclage des déchets organiques $(19,52,57)$. Il a été démontré que les asticots de $M$. domestica (1,5 gr d'œufs pour $1 \mathrm{~kg}$ de substrat) pouvaient réduire de $43 \%$ le substrat de développement composé à $70 \%$ de déchets organiques (52). Le résidu d'élevage peut ensuite être utilisé comme un bio fertilisant de qualité pour améliorer la qualité des sols, augmenter le rendement des cultures et réduire l'utilisation d'engrais chimiques $(16,20,21$, $22,69,71)$. Les teneurs en oligoéléments des résidus produits à partir des différents substrats de production d'asticots ont été comparés (15). Les substrats produits via la dégradation des asticots sont susceptibles d'être utilisés en agriculture (tableau 3). Néanmoins, peu d'études sont réalisées à ce jour dans ce domaine. 
Techniques de production d'asticots de mouches domestiques (Musca domestica L...

Tableau 3 : Oligoéléments contenus dans les différents fumiers biodégradés par les asticots.

\begin{tabular}{|l|l|l|l|l|l|l|}
\hline $\begin{array}{l}\text { Type de } \\
\text { fumier } \\
\text { dégradé }\end{array}$ & $\begin{array}{l}\text { Carbone } \\
\text { organique }(\mathrm{g} \\
\left.\mathrm{kg}^{-1}\right)\end{array}$ & $\begin{array}{l}\text { Azote total } \\
\left.(\mathrm{g} \mathrm{kg})^{-1}\right)\end{array}$ & $\begin{array}{l}\text { Phosphore } \\
\text { total }\left(\mathrm{g} \mathrm{kg}^{-1}\right)\end{array}$ & $\begin{array}{l}\text { Potassium } \\
\text { total }\left(\mathrm{g} \mathrm{kg}^{-1}\right)\end{array}$ & $\begin{array}{l}\text { Calcium } \\
\text { total }\left(\mathrm{g} \mathrm{kg}^{-1}\right)\end{array}$ & $\begin{array}{l}\text { Magnésium } \\
\text { total }\left(\mathrm{g} \mathrm{kg}^{-1}\right)\end{array}$ \\
\hline Porc & $205,0 \pm 0,9$ & $16,9 \pm 0,4$ & $10,3 \pm 0,0$ & $6,0 \pm 0,4$ & $0,03 \pm 0,01$ & $0,07 \pm 0,00$ \\
\hline Volaille & $298,4 \pm 0,5$ & $18,5 \pm 0,3$ & $25,3 \pm 0,0$ & $22,6 \pm 0,6$ & $0,32 \pm 0,00$ & $0,08 \pm 0,00$ \\
\hline $\begin{array}{l}\text { Volaille et } \\
\text { mouton }\end{array}$ & $204,6 \pm 0,4$ & $10,6 \pm 0,3$ & $13,7 \pm 0,0$ & $11,5 \pm 0,3$ & $0,23 \pm 0,01$ & $0,58 \pm 0,01$ \\
\hline $\begin{array}{l}\text { Volaille et } \\
\text { vache }\end{array}$ & $152,2 \pm 1,7$ & $8,8 \pm 0,3$ & $10,7 \pm 0,3$ & $2,5 \pm 0,4$ & $0,25 \pm 0,00$ & $0,42 \pm 0,001$ \\
\hline $\begin{array}{l}\text { Volaille et } \\
\text { porc }\end{array}$ & $340,2 \pm 0,6$ & $18,7 \pm 0,2$ & $18,7 \pm 0,2$ & $9,7 \pm 0,2$ & $0,08 \pm 0,01$ & $0,133 \pm 0,02$ \\
\hline
\end{tabular}

Une étude réalisée au Burkina Faso sur deux années comparant différents amendements seuls ou en mélange (litière de volailles, résidus de production d'asticots, urée et amendements minéraux) sur la culture du maïs a démontré que les traitements contenant le résidu de production d'asticots avaient des meilleurs rendements en graine et en paille que les autres traitements (21). Les résidus de la production des asticots pourraient donc être combinés avec d'autres substrats organiques dans les exploitations agricoles avec la perspective de réduire l'utilisation d'engrais $(6,33,42)$.

\section{Conclusions}

La recherche de protéines alternatives et durables pour l'alimentation de la volaille est une question d'importance majeure qui nécessite des solutions viables à court terme. L'introduction des asticots dans l'alimentation des volailles étant fortement suggérée, cette étude permet une meilleure connaissance des techniques de production d'asticots, de la diversité des substrats susceptible d'être utilisé pour l'élevage des mouches ainsi que la productivité de la biomasse larvaire de certains substrats de production. Cette étude vient de ce fait enrichir la documentation sur l'alimentation des monogastriques à base des asticots en apportant des informations précises afin d'optimiser davantage la production avicole dans l'aviculture moderne et villageoise.

La diversité des substrats organiques, donnent un large choix pour les paysans sur le type de substrats qu'ils peuvent avoir à leur disposition en vue de produire des asticots et contribuer de ce fait à l'élevage durable qui n'est pas encore d'actualité au Niger. Cependant pour une production d'asticots au niveau paysan, nous proposons des substrats à faible coût voir nul (i.e. bouse de vache mélangée avec un autre substrat ou avec des copeaux de bois, fumier de volailles, cadavres d'animaux déchets ménagers) qui doivent être exposés en plein air, contenus dans des seaux ou des pots. 
Les asticots ainsi produits représentent une véritable alternative en source protéique disponible pour la nutrition aviaire. La substitution partielle ou totale de la farine de poisson et de soja dans l'alimentation aviaire par les asticots (vivant, séchés, entiers ou broyés) permet d'obtenir de meilleures performances de poids, de réduction de mortalité et un meilleur rendement économique en alimentation de volailles.

En plus de la production de protéine, le processus d'élevage d'asticots permet d'un coté de recycler les déchets organiques, qui constituent un de nos un défi majeur dans la gestion de l'environnement, et de l'autre ce processus génère un résidu de production qui constitue un véritable biofertilisant pouvant être valorisé en agriculture.

\section{Bibliographie}

1. Abraham, K. O., Richard A., A., et Aihoon, S. (2015). Proximate composition of housefly (Musca domestica) maggots cultured on different substrates as potential feed for Tilapia (Oreochromis niloticus). International Journal of Multidisciplinary Research and Development 2, 102-103.

2. Adesina, M. A., Adejinmi, O. O., Omole, A. J., Fayenuwo, J. A., et Osunkeye, O. (2011). Performance of broilers' finishers fed graded levels of cassava peel -maggot meal- based diet mixtures. Journal of Agriculture, Forestry and the Social Sciences 9, 226-231.

3. Adewolu, M. A., Ikenweiwe, N. B., et Mulero, S. M. (2010). Evaluation of an animal protein mixture as a replacement for fishmeal in practical diets for fingerlings of Clarias gariepinus (Burchell, 1822). Israeli Journal of Aquaculture 62, 237-244.

4. Agbohessou P.S., Syaghalirwa N.M., Armel G., Rudy C.M., Lil-Marlys W.L., Valérie C., Jérôme L., Giorgia P., Frédéric F., Philippe A. L., Patrick K. (2021). Efficiency of fatty acidenriched Dipteran-based meals on zootechnical, digestive, nutritional and immunological parameters of Nile tilapia juveniles. Aquaculture Nutrition 00,1-17

5. Agodokpessi, B. J. (2016). Performances zootechniques des dindonneaux nourris à base de farine d'asticots. Tropicultura 34(3), 253-261.

6. Akanza, K. P., Sanogo, S. and N’Da H. A. (2016). Influence combinée des fumures organique et minérale sur la nutrition et le rendement du maïs : impact sur le diagnostic des carences du sol. Tropicultura 34(2), 208-220.

7. Akpodiete, O. J., Ologhobo, A., et Oluyemi, J. (1997). Production and nutritive value of housefly maggot meal on three substrates of poultry faeces. Journal of Applied Animal Research 12, 101-106.

8. Aniebo, A., et Owen, O. (2010). Effects of age and method of drying on the proximate composition of housefly larvae (Musca domestica Lineaus) meal. Pakistan journal of Nutrition 9, 485-487.

9. Aniebo, A. O., Erondu, E. S., et Owen, O. J. (2008). Proximate composition of housefly larvae (Musca domestica) meal generated from mixture of cattle blood and wheat bran. Livestock Research for Rural Development 20(12), 1-5. 
Techniques de production d'asticots de mouches domestiques (Musca domestica L...

10. Arong, G. A., et Eyo, V. O. (2017). Evaluation of house fly (Musca domestica) maggot meal and termite (Macrotermes subhyalinus) meal as supplementary feed for African catfish Clarias gariepinus (Burchell, 1822) International Journal of Entomology and Nematology 3(1), 043-050.

11. Atteh, J. O., et Ologbenla, F. D. (1993). Replacement of fish meal with maggots in broiler diets: Effects on performance and nutrient retention. Nigerian Journal of Animal Production 20, 44-49.

12. Awoniyi,T.A.M., Adetuyi,F.C. et Akinyosasoye,F.A. (2004). Microbiological investigation of maggot meal stored for use as lifvestock feed component. Journal of Food Agriculture and Environment 2, 104-106

13. Bouafou, K. G. M., Kouame, K. G., Amoikon, K. E., et Offoumou, A. M. (2006). Potentiel pour la production d'asticots sur des sous-produits en Côte d'Ivoire. Tropicultura 24, 157-161.

14. Bouafou, K.G.M., Zannou-Tchoko, V., Konan., B., A., et Kouame, K., G. (2008). Etude de la valeur nutritionnelle de la farine d'asticots séchés chez le rat en croissance. Revue Ivoirienne des Sciences et Technologie 12, 215-225.

15. Bloukounon-Goubalan A.Y, Saïdou A., Noël O., Guillaume L.A., Attanda M.I., Victor A.C., and Kenis M. (2019). Decomposition and nutrient release pattern of animal manures biodegraded by fly larvae in Acrisols. Canadian Journal of Soil Science 99, 60-69

16. Bloukounon-Goubalan A.Y., Saïdou A., Victor A. Clottey, Achille C Chrysostom., A.M., Kenis M. and Mensah G.A. (2017). Typology of organic residues attracting flies and their utilization in the agricultural sector in southern Benin. Intenational Journal of Biological Chemical Science. 11(6), 2560-2572

17. Caparros M.R., Alabi T., Larreché S., Louxinger A., Haubruge É. et Frédéric F. (2015). Risques et valorisation des insectes dans l'alimentation humaine et animale. Annales de la Société entomologique de France 51, 215-258

18. Charlton, A.J., Dickinson, M., Wakefield, M.E., Fitches, E., Kenis, M., Han, R., Zhu, F., Kone, N., Grant, M., Devic, E., Bruggeman, G., Prior, R. and Smith, R. (2015). Exploring the chemical safety of fly larvae as a source of protein for animal feed. Journal of Insects as Food and Feed 1, 7-16.

19. Čičková, H., Pastor, B., Kozánek, M., Martínez-Sánchez, A., Rojo, S., et Takáč, P. (2012). Biodegradation of Pig Manure by the Housefly, Musca domestica: A Viable Ecological Strategy for Pig Manure Management. PLOS ONE 7(3), 32798.

20. Coulibaly, K., Sankara, F., Pousga, S., Nacoulma, P. J. and Nacro, H. B. (2018). Pratiques avicoles et gestion de la fertilité des sols dans les exploitations agricoles de l'Ouest du Burkina Faso. Journal of Applied Biosci-ences 127, 12770-12784.

21. Coulibaly, K*, Sankara F., Pousga S., Philippe J.N., Marc B.S., and Hassan B.(2020) Effects of poultry litter and the residues of maggot's production on chemical fertility of a lixisol and maize (Zea mays L.) yield in western of Burkina Faso. Nigerian Jouranal of Soil 
Science 30(2), 95-102

22. Coulibaly, K*, Fernand Sankara, Salimata Pousga, Philippe J. Nacoulma, Marc B. Somé, and Hassan B.N. (2020). On station maggot production using poultry litter as substrate: assessment on the quantity and the chemical quality of the litter before and after maggot production in Burkina Faso. Intenational Journal of Biological Chemical Science 14(5), 1689-1697

23. Cortes Ortiz, J. A., Ruiz, A. T., Morales-Ramos, J. A., Thomas, M., Rojas, M. G., Tomberlin, J. K., Yi, L., Han, R., Giroud, L., et Jullien, R. L. (2016). Insect Mass Production Technologies. In Insects as Sustainable Food Ingredients. Elsevier 6, 153-201.

24. Diener, S., Zurbrügg, C., Tockner, K. (2015). Bioaccumulation of heavy metals in the black soldier fly, Hermetia illucens 1 and effects on its life cycle. J. Insects Food Feed 1, 261-270.

25. Djissou, A. S. M., Tossavi, E. C., Vodounnou, J. D., Toguyeni, A., et Fiogbe, E. D. (2015). Valorization of agro-alimentary waste for a production of maggots like source of proteins in the animal feeds. International Journal of Agronomy and Agriculture Research 7(6), 42-46.

26. Ekoue, S. E., et Hadzi, Y. A. (2000). Production d'asticots comme source de protéines pour jeunes volailles au Togo-Observations préliminaires. Tropicultura 18(4), 2012-2014.

27. Ezewudo, B. I., Monebi, C. O., et Ugwumba, A. A. A. (2015). Production and utilization of Musca domestica maggots in the diet of Oreochromis niloticus (Linnaeus, 1758) fingerlings. academic Journals 10(23), 2363-2371.

28. Fasakin, E. A., Balogun, A. M., et Ajayi, O. O. (2003). Evaluation of full-fat and defatted maggot meals in the feeding of clariid catfish Clarias gariepinus fingerlings. Aquaculture Researche 34(9), 733-738.

29. FAO. (2019). Passerelle sur l'aviculture et les produits avicoles.

30. Fédération pour l'agriculture et la ruralité dans le monde (FARM). (2008). Les potentialités agricoles de l'Afrique de l'ouest.

31. Gao, M., Lin, Y., Ze Shi, G., Li, H.H., Yang, Z.B., Xu, X.X., Xian, J. R., Yang, Y. X., et Cheng, Z. (2019). Bioaccumulation and health risk assessments of trace elements in housefly (Musca domestica L.) larvae fed with food wastes. Science of the Total Environment $\mathbf{6 8 2}$, 485-493.

32. Greenberg, B. (1973). Flies and diseases. Prince-ton, Princeton University Press

33. Habib, M. A., Khatun, R. and Hossen, M. S. (2015) Impact of organic wastes on soil environment and yield of T-aman rice. Progressive Agriculture 26(2), 122-128.

34. Hardouin, J., et Mahoux, G. (2003). Zootechnie d'insectes-Elevage et utilisation au bénéfice de l'homme et de certains animaux. Bureau pour l'Echange de la distribution de l'information sur le Mini-Elevage 164, 779-3642.

35. Heuzé V., Tran G. (2015). Housefly maggot meal. Feedipedia, a programme by INRA, 
Techniques de production d'asticots de mouches domestiques (Musca domestica L...

CIRAD, AFZ and FAO. https://www.feedipedia.org/node/671 Last updated on October 21, 2015,

36. Holt, P.S., Geden, C.J., Moore, R.W. and Gast, R.K. (2007). Isolation of Salmonella enterica serovar enteritidis from Houseflies. Ap-plied and Environmental Microbiolo-gy, 6030-6035.

37. Hussein, M., Pillai, V. V., Goddard, J. M., Park, H. G., Kothapalli, K. S., Ross, D. A., Ketterings, Q. M., Brenna, J. T., Milstein, M. B., Marquis, H., Johnson, P. A., Nyrop, J. P.,et Selvaraj, V. (2017). Sustainable production of housefly (Musca domestica) larvae as a protein-rich feed ingredient by utilizing cattle manure. PLOS ONE 12(2), e0171708.

38. Hwangbo, J., Hong, E. C., Jan, A., Kang, H. K., Oh, J. S., Kim, B. W., et Park, B. S. (2009). Utilization of house fly-maggots, a feed supplement in the production of broiler chickens. Journal of Environmental Biology. 4(30), 609-614.

39. Janssen, R. H., Vincken, J.-P., van den Broek, L. A. M., Fogliano, V., et Lakemond, C. M. M. (2017). Nitrogen-to-protein conversion factors for three edible insects: Tenebrio molitor, Alphitobius diaperinus, and Hermetia illucens. Journal of Agricultural and Food Chemistry 65(11), 2275-2278.

40. Kenis, M., Koné, N., Chrysostome, C. A. AM., Devic, E., Koko, G. K. D., Clottey, V. A., Nacambo, S., et Mensah, G. A. (2014b). Insects used for animal feed in West Africa. Entomologia 2, 107-114.

41. Koné, N., Sylla, M., Nacambo, S., et Kenis, M. (2017). Production of house fly larvae for animal feed through natural oviposition. Journal of Insects as Food and Feed 3(3), 177-186

42. Koulibaly, B., Dakuo, D., Ouattara, A., Traoré, O., Lompo, F., Zombré, P. N. and YaoKouamé A. (2015). Effets de l'association du compost et de la fumure minérale sur la productivité d'un système de culture à base de cotonnier et de maïs au Burkina Faso. Tropicultura 33 (2), 125-134.

43. Lei, C.L., Zhong, C.Z., Zong, L.B., Niu, C.Y., Jiang, Y. and Song. (1998). Evaluation of the health function of fly-maggot nourishing active powder. Journal of Guiyang Medical College 29, 287-289

44. Li X., Samad Rahimnejad, Ling Wang, Kangle Lu, Kai Song, Chunxiao Zhang.(2019). Substituting fish meal with housefly (Musca domestica) maggot meal in diets for bullfrog Rana (Lithobates) catesbeiana: Effects on growth, digestive enzymes activity, antioxidant capacity and gut health Aquaculture 499, 295-305

45. Makkar, H. P., Tran, G., Heuzé, V., et Ankers, P. (2014). State-of-the-art on use of insects as animal feed. Animal Feed Science and Technology. 197, 1-33.

46. Malik, A., Neena, S., et Santosh, A. (2007). House fly (Musca domestica): A review of control strategies for a challenging pest. Journal of Environmental Science and Health, 42, 453-469. 
47. Mensah, G. A., Pomalégn, S. C. B., Koudjo, A. L., Cakpov, J. C. G., Adjahoutonon, K. Y.K. B., et Agoundo, A. (2007). Farine d'asticots de mouche, une source de protéines bien valorisée dans l'alimentation des canards de barbarie. Communication au 1er Colloque de l’UAC des Sciences et Cultures à Abomey-Calavi (Bénin) du 24 au 29/06/2007. Atelier III : Sciences Naturelles et Agronomiques.

48. Moula, N., \& Detilleux, J. (2019). A Meta-Analysis of the Effects of Insects in Feed on Poultry Growth Performances. Animals 9, 1-13.

49. Mpoame, M., Téguia, A., et Nguemfo, E. L. (2004). Essai comparé de production d'asticot dans les fientes de poule et dans la bouse de vache. Tropicultura 22(2), 84-87.

50. Ngoen-klan, R., Moophayak, T., Klong-klaew, K., Prangkio, C., Irvine, K. N., Sukontason, K., \& Somboon, P. (2011). Do climatic and physical factors affect populations of the blow fly Chrysomya megacephala and house fly Musca domestica. Parasitology Research 109(5), 1279-1292.

51. Nkegbe, E.K., Adu-Aboagye, G., Affedzie, O.S., Nacambo, S., Boafo, A. B., Kenis, M., and Wallace, P. (2018) Potential health and safety issues in the small-scale production of fly larvae for animal feed - a review. Ghanaian Journal of Animal Science 9, 1-10.

52. Niu, Y., Heng, D., Yao, B., Cai, Z., Zhao, Z., Wu, S., Cong, P., et Yang, D. (2017). A novel bioconversion for value-added products from food wast using M. domestica. Waste Management 61, 455-460.

53. Odesanya, B. O., Ajayi, S. O., Agbaogun, B. K. O., et Okuneye, B. (2011). Comparative Evaluation of Nutritive Value of Maggots. International Journal of Scientific et Engineering Research 2(11), 1-5.

54. Ogunji, J., Pagel, T., Schulz, C., Kloas, W. (2009). Apparent digestibility coefficient of housefly maggot meal (magmeal) for Nile tilapia (Oreochromis niloticus L.) and carp (Cyprinus carpio). Asian Fisheries Science 22, 1095-1105.

55. Ogunji, J. O., Kloas, W., Wirth, M., Schulz, C., Rennert, B. (2008). Housefly maggot meal (magmeal) as a protein source for Oreochromis niloticus L. Asian Fisheries Science 21, 319-331.

56. Okah, U., et Onwujiariri, E. B. (2012). Performance of finisher broiler chickens fed maggot meal as a replacement for fish meal. Journal of Agriculture and Technology $\mathbf{8}$, 471-477.

57. Pastor, Berta, Cickova, H., Kozanek, M., Martinez-Sanchez, A., Takac, P., et Rojo, S. (2011). Effect of the size of the pupae, adult diet, oviposition substrate and adult population density on egg production in Musca domestica (Diptera: Muscidae). European Journal of Entomology 108(4), 587-596.

58. Pastor B, Velasquez Y, Gobbi P, Rojo S. 2015. Conversion of organic wastes into fly larval biomass: bottlenecks and challenges. Journal of Insects as Food and Feed, 1, 179-193

59. Pieterse, E., et Pretorius, Q. (2014). Nutritional evaluation of dried larvae and pupae meal 
Techniques de production d'asticots de mouches domestiques (Musca domestica L...

of the housefly (Musca domestica) using chemical- and broiler-based biological assays. Animal Production Science 54(3), 347.

60. Pomalégni, S. C. B., Gbemavo, D. S. J. C., Kpadé, C. P., Kenis, M., et Mensah, G. A. (2017). Traditional use of fly larvae by small poultry farmers in Benin. Journal of Insects as Food and Feed 3(3), 187-192.

61. Réseau national des Chambres d'Agriculture au Niger (RECA). (2016). Guide de l'aviculture au Niger.

62. Rumpold BA, Schlüter OK. (2013). Nutritional composition and safety aspects of edible insects. Molecular. Nutrition \& Food Research. 57, 802-823.

63. Sanou, A. G., Sankara, F., Pousga, S., Coulibaly, K., Nacoulma, J. P., Ouedraogo, I., Nacro, S., Kenis, M., Sanon, A., \& Somda, I. (2019). Production de masse de larves de Musca domestica L. (Diptera: Muscidae) pour l'aviculture au Burkina Faso: Analyse des facteurs déterminants en oviposition naturelle. Journal of Applied Biosciences, 134(1), 13689.

64. Sogbesan, A. O., Ajuonu, N., Mussa, B. O., et Adewole, A. (2006). Harvesting technique and evaluation maggot meals as animal dietary protein source for Heteoclarias in outdoor concrete tanks. World Journal Agricultural Science 2(4), 394-402.

65. Teguia, A., Mpoame, M. and Mba, J.A.O. (2002). The production performance of broiler birds as affected by the replacement of fish meal by maggot meal in the starter and finisher diets. Tropicultura, 20, 187-192.

66. Tendonkeng, F., Miégoué, E., Lemoufouet, J., Mouchili, M., Matimuini, N. F., Mboko, A. V., Zogang, B. F., Mweugang, N. N., Zougou, T. G., Boukila, B., et Pamo, T. E. (2017). Production et composition chimique des asticots en fonction du type de substrat. Livestock research for rural development 29(4), 30.

67. Tobin, P. C., et Pitts, C. W. (2002). Geostatistical analysis and the impact of moisture on the spatial and temporal distribution of larval Musca domestica (Diptera: Muscidae). Environmental Entomology 31(2), 273-280.

68. Van Huis, A., Van Itterbeeck, j., Klunder, H., Martens, E., Halloran, A., Muir, G., et Vantomme, P. (2013). Edible insects: Future prospects for food and feed security. Food and Agriculture Organization of the United Nations 171, 187.

69. Wang, H., Wang S., Li H., Wang B., Zhou Q., Zhang X., Li J., Zhang, Z. (2016). Decomposition and humification of dissolved organic matter in swine manure during housefly larvae composting. Waste Management and Research, 34(5), 465-473.

70. Yang, S., Li, Q., Gao, Y., Zheng, L., \& Liu, Z. (2014). Biodiesel production from swine manure via housefly larvae (Musca domestica L.). Renewable energy 66, 222-224.

71. Zhang, Z., Wang H., Zhu J., Suneethi S., Zheng, J. (2012). Swine manure vermicomposting via housefly larvae (Musca domestica): The dynamics of biochemical and microbial features. Bioresource Technology, 118, 563-571. 
Tropicultura 2295-8010 Volume 39 (2021) Numéro 2, 1813

\section{Notes}

PDF généré automatiquement le 2022-02-11 23:21:26

Url de l'article : https://popups.uliege.be/2295-8010/index.php?id=1813 